\title{
CHEMOSPHERE
}

\section{Estimation of methane and nitrous oxide emission from animal production sector in Taiwan during 1990-2000}

\author{
Shang-Shyng Yang ${ }^{\mathrm{a}, \mathrm{b}, *}$, Chung-Ming Liu ${ }^{\mathrm{c}}$, Yen-Lan Liu ${ }^{\mathrm{c}}$ \\ a Department of Agricultural Chemistry, National Taiwan University, Taipei 10617, Taiwan \\ ${ }^{\mathrm{b}}$ Graduate Institute of Biotechnology, National Pingtung University of Science and Technology, Pingtung 91201, Taiwan \\ ${ }^{\mathrm{c}}$ Department of Atmospheric Sciences, National Taiwan University, Taipei 10617, Taiwan
}

\begin{abstract}
To investigate the greenhouse gases emissions from the feeding and waste management of livestock and poultry, methane and nitrous oxide emissions were estimated from the local measurement and IPCC guidelines during 19902000 in Taiwan. Hog is the major livestock and is followed by goat and cattle, while chicken is the major poultry and is followed by duck and geese. Methane emission from enteric fermentation of livestock was $30.9 \mathrm{Gg}$ in 1990, increased to $39.3 \mathrm{Gg}$ in 1996, and then decreased gradually to $34.9 \mathrm{Gg}$ in 2000 . Methane emission from the waste management was $48.5 \mathrm{Gg}$ in 1990 , reached the peak value of $60.7 \mathrm{Gg}$ in 1996, and then declined to $43.3 \mathrm{Gg}$ in 2000 . In the case of poultry, annual methane emission from enteric fermentation and waste management was 30.6-44.1 ton, and 8.7-13.2 Gg, respectively. Nitrous oxide emission from waste management of livestock was 0.78 ton in 1990 , increased to 0.86 ton in 1996, and then decreased to 0.65 ton in 2000 . Nitrous oxide emission from waste management of poultry was higher than that of livestock with 1.11 ton in 1990, 1.68 ton in 1999, and 1.65 ton in 2000. There is an urgent need to reduce methane emission from enteric fermentation and recover methane from anaerobic waste treatment for energy in livestock and poultry feeding in Taiwan.
\end{abstract}

(c) 2003 Elsevier Ltd. All rights reserved.

Keywords: Animal feeding; Enteric fermentation; Methane; Nitrous oxide; Waste management

\section{Introduction}

Global warming induced by increasing greenhouse gases concentrations in the atmosphere is a matter of great environmental concern. Carbon dioxide is the principal greenhouse gas, followed by methane and nitrous oxide in that order. Methane is mostly produced biologically by methanogenic archaea in anaerobic environments. Flooded paddy, enteric fermentation, animal waste management, agricultural waste burning,

\footnotetext{
${ }^{*}$ Corresponding author. Address: Department of Agricultural Chemistry, National Taiwan University, Taipei 10617, Taiwan. Tel.: +886-2-2362-1519; fax: +886-2-2367-9827.

E-mail address: ssyang@ccms.ntu.edu.tw (S.-S. Yang).
}

savannah burning, landfill, sewage treatment, natural wetland and sediment are considered the major sources of methane emissions (Cicerone and Oremland, 1988; Khalil et al., 1991; Lindau et al., 1993; Liu et al., 1996; Yang, 1998; Yang and Chang, 2001; Yang et al., in press).

Methane is a natural by-product of animal digestion through a process referred to as enteric fermentation. Ruminant animals such as cattle, buffalo, sheep and goat are the high sources of methane, whereas the nonruminant animals such as hog, poultry and horse have much smaller methane emission per animal. The amount of methane produced depends on the type of animal, the amount and the kind of feed it consumes (Kinsman et al., 1995; Lee et al., 2001). In addition, methane emission from animal waste management also contributed to high amount. The anaerobic waste disposal system and the large population of hog explain the large 
amount of methane release (Francisco, 1997; Yang, 1997).

The release of nitrous oxide is increasing in recent years due to intensive agricultural practices and the application of nitrogen fertilizer (Isermann, 1994; Yang et al., in press). Nitrous oxide emission in this section pertains to those releases before the animal wastes are added to soil as fertilizer. These include emission from waste management system other than pasture range and paddock. Nitrous oxide emission is calculated based on animal population, amount of nitrogen excretion, system of waste management and emission factor.

Estimates on greenhouse gas emission from animal feeding and waste management are based on countryspecific emission factors, and when the local data were unavailable, the emission factors recommended by IPCC (1997) guidelines were used.

\section{Methodology and data}

\subsection{Livestock and poultry production}

The livestock and poultry productions in Taiwan from 1990 to 2000 are adapted from the Taiwan Agriculture Yearbook from 1991 to 2001 (Department of Agriculture and Forest, 1991-2000; COA/ROC, 2001).

\subsection{Animal waste production}

The amount of waste production of each head depends on variety, feed composition, feeding method, growth stage and management. Therefore, the value is counted by the average of each species. Each head of 60 $\mathrm{kg}$ hog daily secretes $2.0 \mathrm{~kg}$ feces and $3.75 \mathrm{~kg}$ urine with free or fix-feeding methods. Each head daily secretes $27.5 \mathrm{~kg}$ feces and $13.5 \mathrm{~kg}$ urine for $500 \mathrm{~kg}$ body weight of cattle, $0.75 \mathrm{~kg}$ feces and $1.75 \mathrm{~kg}$ urine for $35 \mathrm{~kg}$ body weight of goat. The poultry daily secretes $0.14-0.15 \mathrm{~kg}$ waste from chicken, $0.16 \mathrm{~kg}$ waste from duck, $0.32 \mathrm{~kg}$ waste from geese and $0.33 \mathrm{~kg}$ waste from turkey (Yang et al., 1991, 1996, 2003).

\subsection{Methane emission factor}

Methane emission factors of enteric fermentation and waste management of livestock and poultry are listed in Table 1. Methane emission factors of enteric fermentations were measured in broiler and colorful broiler by Huang and Wang (2000) and in Holstein by Lee et al. (2000). Methane emission factor in hog waste management was calculated by Lin et al. (1989). Other factors were as recommended by IPCC (1997) guidelines.

\subsection{Nitrous oxide emission factor}

Nitrous oxide emission factors of enteric fermentation and waste management of livestock and poultry are also listed in Table 1. All of them were adapted from the IPCC (1997) guidelines. Waste management includes anaerobic lagoon, anaerobic digestion, daily spread, solid storage and composting, open field feeding and others.

\section{Results and discussion}

\subsection{Methane emission from enteric fermentation}

Domestic animal feeding increased sharply in the past 20 years due to policy encouragement, feeding technology improvement and market requirement. Hog, the major domestic livestock in Taiwan, accounted for $93.9 \%$ of total livestock feeding in 1990 and $93.3 \%$ in 2000. Goat and cattle followed hog. Chicken accounted for $84.6 \%$ of total poultry feeding in 1990 and up to $91.2 \%$ in 2000 . Heads of livestock on farms at the yearend and heads of slaughtered poultry are presented in Table 2. Hog heads reached the maximum of 10698366 in 1996, decreased dramatically to 7966887 in 1997 because of the foot and mouth disease infection, and then increased slightly after 1999. Heads of buffalo, yellow and hybrid cattle, rabbit, deer and turkey decreased gradually from 1990 to 2000, whereas heads of Holstein, goat, milk goat, chicken and geese increased gradually due to domestic demand.

Methane emission factor of enteric fermentation measured in local and recommended by IPCC guidelines is shown in Table 1. In Taiwan, methane emission factor of enteric fermentation was $8.482 \times 10^{-5} \mathrm{~kg} / \mathrm{head}$ in each life cycle of colorful broiler and $1.587 \times 10^{-5} \mathrm{~kg} /$ head in each life cycle of broiler (Huang and Wang, 2000). Lee et al. (2000) indicated that annual methane emission from enteric fermentation in Holstein lactation cow (body weight $611 \pm 56 \mathrm{~kg}$ ) depended on feed, $137.6 \mathrm{~kg}$ with corn silage, $151.5 \mathrm{~kg}$ with Napier grass silage and $161.3 \mathrm{~kg}$ with Pangola grass haylage; annual carbon dioxide emissions from enteric fermentation were 3760,5366 and $4051 \mathrm{~kg}$, respectively. However, annual methane emissions from enteric fermentation was 91.3 $\mathrm{kg}$ with corn silage, $69.0 \mathrm{~kg}$ with Napier grass silage and $88.7 \mathrm{~kg}$ with Pangola grass haylage in Holstein dry cow (average body weight $425 \mathrm{~kg}$ ) and were 61.0, 59.9 and $70.5 \mathrm{~kg}$ in growing Heifer (average body weight $275 \mathrm{~kg}$ ), respectively. Annual carbon dioxide emissions was 3186 $\mathrm{kg}$ with corn silage, $2562 \mathrm{~kg}$ with Napier grass silage and $2686 \mathrm{~kg}$ with Pangola grass haylage in Holstein dry cow and 1902,1781 and $2051 \mathrm{~kg}$ in growing Heifer, respectively (Lee et al., 2001). The average of annual methane emission from enteric fermentation in Holstein lactation 
Table 1

Emission coefficient of greenhouse gases in enteric fermentation and waste management

\begin{tabular}{|c|c|c|c|c|c|c|c|}
\hline \multirow[t]{2}{*}{ Item } & \multicolumn{2}{|c|}{$\begin{array}{l}\mathrm{CH}_{4} \text { emission coefficient } \\
(\mathrm{kg} / \mathrm{h} \text { ead/year })\end{array}$} & \multicolumn{5}{|c|}{$\mathrm{N}_{2} \mathrm{O}$ emission coefficient (mg/head/year) } \\
\hline & $\begin{array}{l}\text { Enteric } \\
\text { fermentation }\end{array}$ & $\begin{array}{l}\text { Waste } \\
\text { management }\end{array}$ & $\begin{array}{l}\text { Anaerobic } \\
\text { lagoon }\end{array}$ & $\begin{array}{l}\text { Anaerobic } \\
\text { digestion }\end{array}$ & Daily spread & $\begin{array}{l}\text { Solid storage } \\
\text { (composting) }\end{array}$ & $\begin{array}{l}\text { Open field } \\
\text { feeding }\end{array}$ \\
\hline $\operatorname{Hog}^{\mathrm{a}}$ & 1.5 & $5.475^{\mathrm{b}}$ & 2.08 & 11.2 & 0.24 & 48.0 & - \\
\hline Holstein $^{a}$ & 56.0 & 16.0 & 3.0 & 12.0 & 6.0 & 660.0 & 120.0 \\
\hline Holstein $^{c}$ & 149.47 & - & - & - & - & - & - \\
\hline Buffalo $^{\mathrm{a}}$ & 55.0 & 2.0 & - & - & - & - & - \\
\hline $\begin{array}{l}\text { Yellow and } \\
\text { hybrid cattle }\end{array}$ & 44.0 & 1.0 & 0.0 & 4.0 & 4.0 & 400.0 & 240.0 \\
\hline Goat $^{\mathrm{a}}$ & 5.0 & 0.18 & 1.2 & 2.4 & - & 144.0 & - \\
\hline Milk goat $^{\mathrm{d}}$ & 8.0 & 0.28 & 1.92 & 3.84 & - & 230.4 & - \\
\hline Rabbit $^{\mathrm{d}}$ & 0.143 & 0.005 & 0.034 & 0.069 & - & 4.116 & - \\
\hline Deer $^{\mathrm{d}}$ & 5.0 & 0.18 & 1.2 & 2.4 & - & 144.0 & - \\
\hline Horse $^{\mathrm{d}}$ & 18.0 & 2.08 & 0.0 & 4.0 & 4.0 & 400.0 & 240.0 \\
\hline Chicken $^{\mathrm{a}}$ & - & 0.048 & - & - & 0.006 & 11.88 & - \\
\hline Colorful broiler ${ }^{\mathrm{e}, \mathrm{g}}$ & $8.482 \times 10^{-5}$ & - & - & - & - & - & - \\
\hline Broiler $^{\mathrm{e}, \mathrm{g}}$ & $1.587 \times 10^{-5}$ & - & - & - & - & - & - \\
\hline Layer $^{\mathrm{f}, \mathrm{g}}$ & $5.035 \times 10^{-5}$ & - & - & - & - & - & - \\
\hline $\operatorname{Duck}^{\mathrm{f}, \mathrm{g}}$ & $5.239 \times 10^{-5}$ & 0.05 & 0.0 & 0.0 & $6.2 \times 10^{-3}$ & 12.36 & - \\
\hline Geese $^{\mathrm{f}, \mathrm{g}}$ & $1.158 \times 10^{-4}$ & 0.110 & 0.0 & 0.0 & $1.43 \times 10^{-2}$ & 28.44 & - \\
\hline Turkeyf, $\mathrm{g}$ & $1.158 \times 10^{-4}$ & 0.110 & 0.0 & 0.0 & $1.43 \times 10^{-2}$ & 28.44 & - \\
\hline
\end{tabular}

${ }^{\mathrm{a}}$ IPCC (1997).

${ }^{\mathrm{b}}$ Lin et al. (1989).

${ }^{\mathrm{c}}$ Lee et al. (2000).

${ }^{\mathrm{d}}$ The values of milk goat, deer, horse and rabbit were calculated from goat and adjusted by body weight of livestock.

${ }^{\mathrm{e}}$ Huang and Wang (2000).

${ }^{\mathrm{f}}$ The values of layer, duck, geese and turkey were calculated from broiler and colorful broiler and adjusted by body weight of poultry.

${ }^{\mathrm{g}}$ The values were represented in $\mathrm{kg} / \mathrm{head} / \mathrm{life}$ cycle for methane emission coefficient, and $\mathrm{mg} / \mathrm{head} / \mathrm{life}$ cycle for nitrous oxide emission coefficient.

cow, Holstein dry cow and growing Heifer with three feeds was $150.1,116.6$ and $63.8 \mathrm{~kg} / \mathrm{head}$, respectively. These values are 2.68, 2.08 and 1.14 times higher than that recommended by IPCC (1997) guidelines. The average of annual carbon dioxide emission from enteric fermentation in Holstein lactation cow, Holstein dry cow and growing Heifer with three feeds was 4392, 2811 and $1911 \mathrm{~kg}$, respectively. Annual enteric fermentations of methane and nitrous oxide of milk goat, rabbit, deer and horse were calculated from goat and adjusted by the body weight, while annual enteric fermentations of methane and nitrous oxide of layer, duck, geese and turkey were estimated from the average value in broiler and colorful broiler with local measurement and adjusted by the body weight.

Annual heads of livestock were calculated from the heads on farms at the year-end and annual heads of poultry were counted from the heads of slaughter. Methane emission from the enteric fermentation of livestock and poultry in Taiwan is illustrated in Table 3. It was calculated from the heads of livestock and poultry times the emission factors. It was found that methane emission from enteric fermentation of animal feeding increased from $30.83 \mathrm{Gg}$ in 1990 to $39.23 \mathrm{Gg}$ in 1996 , and then decreased to $34.88 \mathrm{Gg}$ in 2000 . Holstein is the largest source of methane emission, contributing $44.0 \%$ of the total enteric fermentation in 1990 and gradually up to $58.5 \%$ in 2000 . Hog contributed $41.7 \%$ of the total enteric fermentation in 1990 and $32.2 \%$ in 2000 . The heads of poultry are numerous, but their body weights are low. In addition, poultry is non-ruminant and non-herbivorous. Therefore, methane emission from enteric fermentation of poultry was below $1 \%$ of the total methane emission from animal feeding sector.

\subsection{Methane emission from waste management}

Feces and urine are separated in livestock, while they are mixed together in poultry. The amount of waste production of each head depends on variety, feeding method, feed formulation, growth stage, managing method and body weight (Hong, 1986a,b; Yang et al., 1991, 1996, 2003). Yang (1994) and Wong et al. (1999) calculated feces production of livestock and poultry in Taiwan, which are presented in Table 4. Annual animal solid waste was 12.5 million ton in 1990 , reached a 
Table 2

Heads of livestock on farms at the year-end and heads of poultry slaughtered in Taiwan

\begin{tabular}{|c|c|c|c|c|c|c|c|c|c|c|c|}
\hline Item & 1990 & 1991 & 1992 & 1993 & 1994 & 1995 & 1996 & 1997 & 1998 & 1999 & 2000 \\
\hline \multicolumn{12}{|c|}{ Livestock on farms $\left(\times 10^{3}\right)$} \\
\hline Hog & 8565 & 10089 & 9754 & 9845 & 10066 & 10509 & 10698 & 7967 & 6539 & 7243 & 7495 \\
\hline Holstein & 91 & 101 & 111 & 117 & 119 & 124 & 125 & 133 & 134 & 136 & 137 \\
\hline Buffalo & 22 & 19 & 17 & 16 & 15 & 13 & 11 & 10 & 9 & 9 & 8 \\
\hline $\begin{array}{l}\text { Yellow and } \\
\text { hybrid cattle }\end{array}$ & 42 & 34 & 31 & 32 & 31 & 28 & 26 & 24 & 23 & 20 & 17 \\
\hline Goat & 173 & 176 & 202 & 294 & 311 & 319 & 310 & 315 & 316 & 237 & 202 \\
\hline Milk goat & 34 & 40 & 45 & 72 & 90 & 112 & 119 & 127 & 129 & 126 & 113 \\
\hline Rabbit & 161 & 139 & 128 & 112 & 87 & 79 & 76 & 55 & 55 & 43 & 40 \\
\hline Deer & 36 & 33 & 30 & 29 & 27 & 24 & 23 & 23 & 22 & 21 & 20 \\
\hline Horse & 0.9 & 0.8 & 0.8 & 0.8 & 0.8 & 0.7 & 0.6 & 0.7 & 0.6 & 06 & 0.8 \\
\hline Total & 9125 & 10630 & 10319 & 10518 & 10746 & 11207 & 11389 & 8654 & 7226 & 7836 & 8033 \\
\hline \multicolumn{12}{|c|}{ Poultry head slaughtered $\left(\times 10^{3}\right)$} \\
\hline Chicken & 226556 & 233971 & 257666 & 288243 & 301914 & 319820 & 345509 & 389966 & 389524 & 385563 & 389770 \\
\hline $\begin{array}{l}\text { Colorful } \\
\text { broiler }\end{array}$ & 135664 & 126692 & 136831 & 147906 & 149933 & 150756 & 164084 & 180072 & 175215 & 175328 & 173627 \\
\hline Broiler & 74415 & 97504 & 104247 & 123161 & 133495 & 149451 & 159983 & 185280 & 189535 & 185077 & 191202 \\
\hline Layer & 16477 & 15775 & 16588 & 17176 & 18486 & 19613 & 21442 & 24614 & 24774 & 25158 & 24948 \\
\hline Duck & 39900 & 36295 & 40558 & 45483 & 40886 & 42580 & 41759 & 41156 & 35719 & 35208 & 34099 \\
\hline Tsaiya & 1631 & 1556 & 1764 & 1943 & 1982 & 2070 & 2131 & 2146 & 2116 & 2049 & 2024 \\
\hline Mule-duck & 38269 & 34739 & 38794 & 43540 & 38904 & 40510 & 39628 & 39010 & 33603 & 33195 & 32075 \\
\hline Geese & 4777 & 4628 & 5683 & 6397 & 8521 & 7744 & 7078 & 7503 & 7955 & 7464 & 6503 \\
\hline Turkey & 758 & 636 & 543 & 521 & 458 & 415 & 398 & 429 & 432 & 488 & 500 \\
\hline Total & 271991 & 275530 & 304450 & 340644 & 351779 & 370559 & 394774 & 439054 & 433630 & 428723 & 430871 \\
\hline
\end{tabular}

Table 3

Estimation of methane emissions from the enteric fermentation of livestock and poultry in Taiwan (ton)

\begin{tabular}{|c|c|c|c|c|c|c|c|c|c|c|c|}
\hline Item & 1990 & 1991 & 1992 & 1993 & 1994 & 1995 & 1996 & 1997 & 1998 & 1999 & 2000 \\
\hline Livestock & 30828.8 & 34081.1 & 34782.3 & 36816.7 & 37442.4 & 38916.8 & 39227.3 & 36108.8 & 34066.7 & 34901.2 & 34879.8 \\
\hline Hog & 12847.9 & 15133.7 & 14631.7 & 14767.4 & 15098.3 & 15762.8 & 16047.6 & 11950.3 & 9807.9 & 10864.8 & 11242.4 \\
\hline Holstein & 13571.6 & 15027.6 & 16309.4 & 17494.4 & 17712.6 & 18588.8 & 18754.2 & 19835.3 & 20034.5 & 20325.5 & 20404.8 \\
\hline Buffalo & 1201.2 & 1024.0 & 914.3 & 906.9 & 820.0 & 708.6 & 616.7 & 528.1 & 470.6 & 505.4 & 427.2 \\
\hline $\begin{array}{l}\text { Yellow and } \\
\text { hybrid cattle }\end{array}$ & 1828.8 & 1482.8 & 1348.8 & 1411.0 & 1357.8 & 1213.4 & 1162.9 & 1059.9 & 1003.5 & 883.3 & 766.4 \\
\hline Goat & 865.0 & 878.8 & 1011.7 & 1471.2 & 1554.0 & 1593.8 & 1547.5 & 1577.0 & 1578.1 & 1186.5 & 1012.5 \\
\hline Milk goat & 270.2 & 316.8 & 363.7 & 576.0 & 723.1 & 894.7 & 952.4 & 1017.2 & 1032.9 & 1006.7 & 900.4 \\
\hline Rabbit & 46.1 & 39.7 & 36.5 & 31.9 & 24.7 & 22.5 & 21.7 & 15.8 & 15.6 & 12.8 & 12.0 \\
\hline Deer & 182.4 & 164.2 & 152.2 & 143.7 & 137.2 & 119.9 & 113.2 & 113.1 & 112.4 & 106.0 & 100.1 \\
\hline Horse & 15.7 & 13.5 & 14.0 & 14.2 & 14.6 & 12.4 & 11.3 & 12.2 & 11.2 & 10.2 & 14.0 \\
\hline Poultry & 31.9 & 30.6 & 33.2 & 36.3 & 36.9 & 37.7 & 40.3 & 44.1 & 43.0 & 42.8 & 42.4 \\
\hline Chicken & 13.5 & 13.1 & 14.1 & 15.4 & 15.8 & 16.2 & 17.5 & 19.5 & 19.1 & 19.1 & 19.0 \\
\hline $\begin{array}{l}\text { Colorful } \\
\text { broiler }\end{array}$ & 11.5 & 10.8 & 11.6 & 12.6 & 12.7 & 12.8 & 13.9 & 15.3 & 14.9 & 14.9 & 14.7 \\
\hline Broiler & 1.2 & 1.6 & 1.7 & 2.0 & 2.1 & 2.4 & 2.5 & 2.9 & 3.0 & 2.9 & 3.0 \\
\hline Layer & 0.8 & 0.8 & 0.8 & 0.9 & 0.9 & 1.0 & 1.1 & 1.2 & 1.3 & 1.3 & 1.3 \\
\hline Duck & 2.1 & 1.9 & 2.1 & 2.4 & 2.1 & 2.2 & 2.2 & 2.2 & 1.9 & 1.9 & 1.8 \\
\hline Tsaiya & 0.1 & 0.1 & 0.1 & 0.1 & 0.1 & 0.1 & 0.1 & 0.1 & 0.1 & 0.1 & 0.1 \\
\hline Mule-duck & 2.0 & 1.8 & 2.0 & 2.3 & 2.0 & 2.1 & 2.1 & 2.0 & 1.8 & 1.7 & 1.7 \\
\hline Geese & 0.6 & 0.6 & 0.7 & 0.7 & 1.0 & 0.9 & 0.8 & 0.9 & 0.9 & 0.9 & 0.8 \\
\hline Turkey & 0.1 & 0.1 & 0.1 & 0.1 & 0.1 & 0.1 & 0.1 & 0.1 & 0.1 & 0.1 & 0.1 \\
\hline Total & 30860.6 & 34111.7 & 34815.4 & 36853.0 & 37479.2 & 38954.5 & 39267.7 & 36152.9 & 34109.7 & 34943.9 & 34922.2 \\
\hline
\end{tabular}


Table 4

Feces production of livestock and poultry in Taiwan

\begin{tabular}{|c|c|c|c|c|c|c|c|c|c|c|c|c|c|}
\hline \multirow[t]{2}{*}{ Item } & \multirow{2}{*}{$\begin{array}{l}\text { Daily } \\
\text { secrete } \\
(\mathrm{kg} / \\
\text { head })\end{array}$} & \multirow{2}{*}{$\begin{array}{l}\text { Annual } \\
\text { amount } \\
\text { (kg/ } \\
\text { head) }\end{array}$} & \multicolumn{11}{|c|}{ Total amount $\left(10^{3}\right.$ ton $)$} \\
\hline & & & 1990 & 1991 & 1992 & 1993 & 1994 & 1995 & 1996 & 1997 & 1998 & 1999 & 2000 \\
\hline $\begin{array}{l}\text { Live- } \\
\text { stock }\end{array}$ & & & 7555 & 8710 & 8548 & 8719 & 8889 & 9251 & 9386 & 7448 & 6408 & 6908 & 7064 \\
\hline Hog & 2.0 & 730 & 6253 & 7365 & 7121 & 7187 & 7348 & 7671 & 7810 & 5816 & 4773 & 5288 & 5471 \\
\hline Holstein & 27.5 & 10038 & 911 & 1009 & 1110 & 1175 & 1190 & 1248 & 1259 & 1332 & 1345 & 1365 & 1370 \\
\hline Buffalo & 13.75 & 5019 & 110 & 93 & 83 & 83 & 75 & 65 & 56 & 48 & 43 & 46 & 39 \\
\hline $\begin{array}{l}\text { Yellow } \\
\text { and } \\
\text { hybrid } \\
\text { cattle }\end{array}$ & 13.75 & 5019 & 209 & 169 & 154 & 161 & 155 & 138 & 133 & 121 & 114 & 101 & 87 \\
\hline Goat & 0.75 & 273.8 & 47 & 48 & 53 & 81 & 85 & 87 & 85 & 86 & 86 & 65 & 55 \\
\hline $\begin{array}{l}\text { Milk } \\
\text { goat }\end{array}$ & 0.75 & 273.8 & 9 & 11 & 12 & 20 & 25 & 31 & 33 & 35 & 35 & 34 & 31 \\
\hline Rabbit & 0.027 & 9.855 & 2 & 2 & 1 & 1 & 1 & 1 & 1 & 1 & 1 & 1 & 1 \\
\hline Deer & 0.75 & 273.8 & 10 & 9 & 8 & 8 & 8 & 7 & 6 & 6 & 6 & 6 & 5 \\
\hline Horse & 13.75 & 5019 & 4 & 4 & 4 & 4 & 4 & 3 & 3 & 3 & 3 & 3 & 4 \\
\hline $\begin{array}{l}\text { Poultry } \\
\text { Chicken }\end{array}$ & & & 4895 & 5010 & 5730 & 5975 & 6277 & 6446 & 6898 & 7223 & 7363 & 7405 & 7252 \\
\hline $\begin{array}{r}\text { Colorful } \\
\text { broiler }\end{array}$ & 0.14 & 51.1 & 1862 & 1869 & 2100 & 2182 & 2184 & 2265 & 2414 & 2482 & 2477 & 2532 & 2367 \\
\hline Broiler & 0.14 & 51.1 & 906 & 984 & 1142 & 1257 & 1392 & 1456 & 1596 & 1770 & 1882 & 1845 & 1816 \\
\hline $\begin{array}{l}\text { Layer } \\
\text { Duck }\end{array}$ & 0.15 & 54.75 & 1249 & 1275 & 1327 & 1370 & 1524 & 1588 & 1756 & 1881 & 1928 & 1964 & 1973 \\
\hline Tsaiya & 0.16 & 58.4 & 128 & 128 & 155 & 165 & 166 & 174 & 169 & 184 & 170 & 169 & 170 \\
\hline $\begin{array}{l}\text { Mule- } \\
\text { duck }\end{array}$ & 0.16 & 58.4 & 492 & 494 & 704 & 613 & 584 & 590 & 589 & 509 & 502 & 514 & 450 \\
\hline Geese & 0.32 & 116.8 & 216 & 226 & 269 & 356 & 400 & 348 & 353 & 371 & 377 & 351 & 446 \\
\hline Turkey & 0.33 & 120.45 & 42 & 34 & 32 & 32 & 26 & 25 & 22 & 25 & 28 & 32 & 30 \\
\hline Total & & & 12451 & 13720 & 14278 & 14694 & 15166 & 15698 & 16283 & 14671 & 13771 & 14313 & 14316 \\
\hline
\end{tabular}

maximal value of 16.3 million ton in 1996, and decreased to 14.3 million ton in 2000 . Hog feces is the major animal waste, accounting for $50.2 \%$ of the total animal waste in $1990,53.7 \%$ in $1991,34.7 \%$ in 1998 , and $38.2 \%$ in 2000. Chicken manure is the next to hog and accounted for $32.3 \%$ of the total animal waste in 1990 , $45.7 \%$ in 1998 , and $43.0 \%$ in 2000 .

Hog (60 kg weight) daily produced 601 gas containing $60-80 \%$ of methane, with the annual methane production of $10.95 \mathrm{~kg}$ (Hong, 1986b). Lin et al. (1989) reported that each head of hog daily produced 401 of wastewater, and generated 31.41 of methane after anaerobic digestion. The average daily methane emission from hog waste management was 211 (equivalent to 15.0 $\mathrm{g}$ methane), and the annual methane emission was 5.475 $\mathrm{kg} /$ head. Methane emission from animal waste management, estimated with local measurement and IPCC recommendation, is listed in Table 5. With IPCC guidelines, methane emission from waste management was 40317 ton in 1990 , had a peak value of 51251 ton in 1996, decreased to 35177 ton in 1998 due to the widespread foot and mouth disease, and then increased gradually to 39033 ton in 2000. Based on the local measurement data, methane emission from waste management was 57176 ton in 1990, had the highest value 72985 ton in 1996, decreased to 51182 ton in 1998, and then increased gradually to 56277 ton in 2000. High methane emission from hog waste management in Taiwan might be due to the high temperature environment which favors the methanogenesis. It showed that hog waste management, the major source of methane emission, accounted for $82.0 \%$ of the total methane emission in $1990,83.9 \%$ in $1991,69.9 \%$ in 1998 , and $72.9 \%$ in 2000. Chicken manure was the next to hog waste, accounting for $5.8 \%$ of the total methane emission in 1991 and $11.3 \%$ in 1998 .

\subsection{Nitrous oxide emission from waste management}

In Taiwan, livestock liquid waste is subjected to anaerobic digestion, followed by aeration or facultative treatment to remove BOD, COD and SS, while the solid waste is used as raw materials for composting. Chao (2001) studied the effect of livestock and poultry compost 
Table 5

Estimation of methane emission from the waste management of livestock and poultry in Taiwan (ton)

\begin{tabular}{|c|c|c|c|c|c|c|c|c|c|c|c|}
\hline Item & 1990 & 1991 & 1992 & 1993 & 1994 & 1995 & 1996 & 1997 & 1998 & 1999 & 2000 \\
\hline Livestock & 48481.9 & 56968.4 & 55296.3 & 55919.3 & 57154.1 & 59672.0 & 60724.2 & 45883.7 & 38082.1 & 41953.3 & 43325.6 \\
\hline Hog & 46894.0 & 55238.0 & 53406.0 & 53901.0 & 55109.0 & 57534.0 & 58573.0 & 43619.0 & 35799.0 & 39656.0 & 41035.0 \\
\hline Holstein & 1452.8 & 1608.6 & 1769.5 & 1872.7 & 1896.1 & 1989.8 & 2007.5 & 2123. 3 & 2144.6 & 2175.7 & 2184.2 \\
\hline Buffalo & 43.8 & 37.2 & 33.3 & 33.0 & 29.8 & 25.8 & 22.4 & 19.2 & 17.1 & 18.4 & 15.5 \\
\hline $\begin{array}{l}\text { Yellow and } \\
\text { hybrid cattle }\end{array}$ & 41.6 & 33.7 & 30.7 & 32.1 & 30.9 & 27.6 & 26.4 & 24.1 & 22.8 & 20.1 & 17.4 \\
\hline Goat & 31.1 & 31.6 & 36.4 & 53.0 & 56.0 & 57.4 & 55.7 & 56.8 & 56.8 & 42.7 & 36.5 \\
\hline Milk goat & 9.5 & 11.1 & 12.7 & 20.2 & 25.3 & 31.3 & 33.3 & 35.6 & 36.2 & 35.2 & 31.5 \\
\hline Rabbit & 0.8 & 0.7 & 0.6 & 0.6 & 0.4 & 0.4 & 0.4 & 0.3 & 0.3 & 0.2 & 0.2 \\
\hline Deer & 6.6 & 5.9 & 5.5 & 5.2 & 4.9 & 4.3 & 4.1 & 4.1 & 4.1 & 3.8 & 3.6 \\
\hline Horse & 1.8 & 1.6 & 1.6 & 1.6 & 1.7 & 1.4 & 1.3 & 1.4 & 1.3 & 1.2 & 1.6 \\
\hline Poultry & 8694.3 & 8905.3 & 10173.2 & 10560.0 & 11067.3 & 11435.0 & 12261.2 & 12845.6 & 13100.2 & 13189.5 & 12951.6 \\
\hline Chicken & 3695.0 & 3797.8 & 4209.2 & 4431.8 & 4695.7 & 4888.2 & 5305.7 & 5643. 1 & 5784.5 & 5832.6 & 5880.6 \\
\hline $\begin{array}{l}\text { Colorful } \\
\text { broiler }\end{array}$ & 1749.0 & 1755.7 & 1972.9 & 2049.5 & 2051.9 & 2127.8 & 2267.2 & 2331. 1 & 2326.4 & 2378.2 & 2223.3 \\
\hline Broiler & 850.6 & 924.1 & 1073.1 & 1180.9 & 1307.9 & 1367.9 & 1498.9 & 1662.9 & 1767.4 & 1732.8 & 1705.7 \\
\hline Layer & 1095.4 & 1118.0 & 1163.2 & 1201.4 & 1335.9 & 1392.6 & 1539.6 & 1649.1 & 1690.7 & 1721.6 & 1729.5 \\
\hline Duck & 531.2 & 533.1 & 736.0 & 665.8 & 642.2 & 654.2 & 648.9 & 593.2 & 575.7 & 582.5 & 564.1 \\
\hline Tsaiya & 110.0 & 109.9 & 132.8 & 141.3 & 142.2 & 148.7 & 144.4 & 157.3 & 145.7 & 144.7 & 145.7 \\
\hline Mule-duck & 421.3 & 423.2 & 603.2 & 524.5 & 500.0 & 505.6 & 504.5 & 435.9 & 430.0 & 437.8 & 385.2 \\
\hline Geese & 203.5 & 212.4 & 253.6 & 335.4 & 377.0 & 327.7 & 332.3 & 349.8 & 354.8 & 330.7 & 288.1 \\
\hline Turkey & 38.4 & 31.2 & 29.4 & 29.5 & 23.7 & 22.4 & 19.8 & 23.2 & 25.2 & 28.8 & 29.5 \\
\hline Total & 57176.2 & 65873.7 & 65469.5 & 66479.2 & 68230.4 & 71107.0 & 72985.3 & 58729.3 & 51182.3 & 55142.9 & 56277.2 \\
\hline
\end{tabular}

Table 6

Estimation of nitrous oxide emission from the waste management of livestock and poultry in Taiwan $(\mathrm{kg})$

\begin{tabular}{|c|c|c|c|c|c|c|c|c|c|c|c|}
\hline Item & 1990 & 1991 & 1992 & 1993 & 1994 & 1995 & 1996 & 1997 & 1998 & 1999 & 2000 \\
\hline Livestock & 780.9 & 776.3 & 765.4 & 796.5 & 816.0 & 850.1 & 860.9 & 698.8 & 611.0 & 641.9 & 646.9 \\
\hline Hog & 626.9 & 620.7 & 600.1 & 605.7 & 619.2 & 646.5 & 658.2 & 490.1 & 402.3 & 445.6 & 461.1 \\
\hline Holstein & 72.7 & 80.5 & 88.6 & 93.8 & 94.9 & 99.6 & 100.5 & 106.3 & 107.4 & 108.9 & 109.3 \\
\hline Buffalo & 14.2 & 12.1 & 10.8 & 10.7 & 9.7 & 8.4 & 7.3 & 6.2 & 5.5 & 6.0 & 5.0 \\
\hline $\begin{array}{l}\text { Yellow and } \\
\text { hybrid cattle }\end{array}$ & 26.9 & 21.8 & 19.9 & 20.8 & 20.0 & 17.9 & 17.1 & 15.6 & 14.8 & 13.0 & 11.3 \\
\hline Goat & 25.5 & 25.9 & 29.9 & 43.4 & 45.9 & 47.1 & 45.7 & 46.6 & 46.6 & 35.0 & 29.9 \\
\hline Milk goat & 8.0 & 9.4 & 10.7 & 17.0 & 21.4 & 26.4 & 28.1 & 30.0 & 30.5 & 29.7 & 26.6 \\
\hline Rabbit & 0.7 & 0.6 & 0.5 & 0.5 & 0.4 & 0.3 & 0.3 & 0.2 & 0.2 & 0.2 & 0.2 \\
\hline Deer & 5.4 & 4.9 & 4.5 & 4.3 & 4.1 & 3.5 & 3.4 & 3.3 & 3.3 & 3.1 & 3.0 \\
\hline Horse & 0.6 & 0.5 & 0.5 & 0.5 & 0.5 & 0.5 & 0.4 & 0.4 & 0.4 & 0.4 & 0.5 \\
\hline Poultry & 1108.9 & 1135.3 & 1297.5 & 1356.5 & 1425.2 & 1462.8 & 1565.4 & 1640.6 & 1673.0 & 1681.3 & 1648.3 \\
\hline Chicken & 915.0 & 940.4 & 1042.3 & 1097.4 & 1162.8 & 1210.5 & 1313.8 & 1397.4 & 1432.4 & 1444.3 & 1401.2 \\
\hline $\begin{array}{l}\text { Colorful } \\
\text { broiler }\end{array}$ & 433.1 & 434.7 & 488.5 & 507.5 & 508.1 & 526.9 & 561.4 & 577.2 & 576.1 & 588.9 & 550.5 \\
\hline Broiler & 210.6 & 228.8 & 265.7 & 292.4 & 323.9 & 338.7 & 371.2 & 411.8 & 437.7 & 429.1 & 422.4 \\
\hline Layer & 271.2 & 276.9 & 288.1 & 297.5 & 330.8 & 344.8 & 381.2 & 408.4 & 418.7 & 426.3 & 428.3 \\
\hline Duck & 131.4 & 131.8 & 182.0 & 164.7 & 158.8 & 161.8 & 160.5 & 146.7 & 142.4 & 144.0 & 131.3 \\
\hline Tsaiya & 27.2 & 27.2 & 32.8 & 35.0 & 35.2 & 36.8 & 35.7 & 38.9 & 36.0 & 35.8 & 36.0 \\
\hline Mule-duck & 104.2 & 104.7 & 149.2 & 129.7 & 123.7 & 125.0 & 124.8 & 107.8 & 106.4 & 108.3 & 95.3 \\
\hline Geese & 52.6 & 55.0 & 65.6 & 86.8 & 97.5 & 84.8 & 86.0 & 90.5 & 91.8 & 85.5 & 108.7 \\
\hline Turkey & 9.9 & 8.1 & 7.6 & 7.6 & 6.1 & 5.8 & 5.1 & 6.0 & 6.5 & 7.5 & 7.1 \\
\hline Total & 1889.8 & 1911.6 & 2062.9 & 2153.0 & 2241.2 & 2313.8 & 2426.3 & 2339.4 & 2284.0 & 2323.2 & 2297.2 \\
\hline
\end{tabular}

on nitrous oxide emission from soils. Application of cow, chicken and hog compost released $1.07-1.28 \%$,
$0.46-0.60 \%$ and $0.79 \%$ of nitrogen as nitrous oxide emission, respectively. There is no local measurement on 
the nitrous oxide emission from waste management. Therefore, nitrous oxide emission from anaerobic digestion, anaerobic lagoon, daily spread, solid storage and composting, open field feeding and other treatments were adapted from the IPCC guidelines (1997).

Nitrification and denitrification of nitrogen compounds in the wastes and in the soils can release nitrous oxide. Nitrous oxide emission from animal waste management increased from $1890 \mathrm{~kg}$ in 1990 to $2426 \mathrm{~kg}$ in 1996 , and then decreased slightly to $2297 \mathrm{~kg}$ in 2000 (Table 6). Chicken waste contributed $48.4 \%$ of the total nitrous oxide emission from animal waste management in 1990 and $61.0 \%$ in 2000 . Hog waste contributed $33.2 \%$ in 1990 and $20.1 \%$ in 2000 .

\section{Conclusion}

Methane and nitrous oxide emissions from enteric fermentation and waste management in Taiwan are summarized in Fig. 1. Methane emission was distinctly higher than that of nitrous oxide in animal feeding sector. Total methane emission amounted to $88.04 \mathrm{Gg}$ in $1990,112.25 \mathrm{Gg}$ in 1996, and $91.20 \mathrm{Gg}$ in 2000. Total nitrous oxide emission was only 1.89 ton in 1990, had

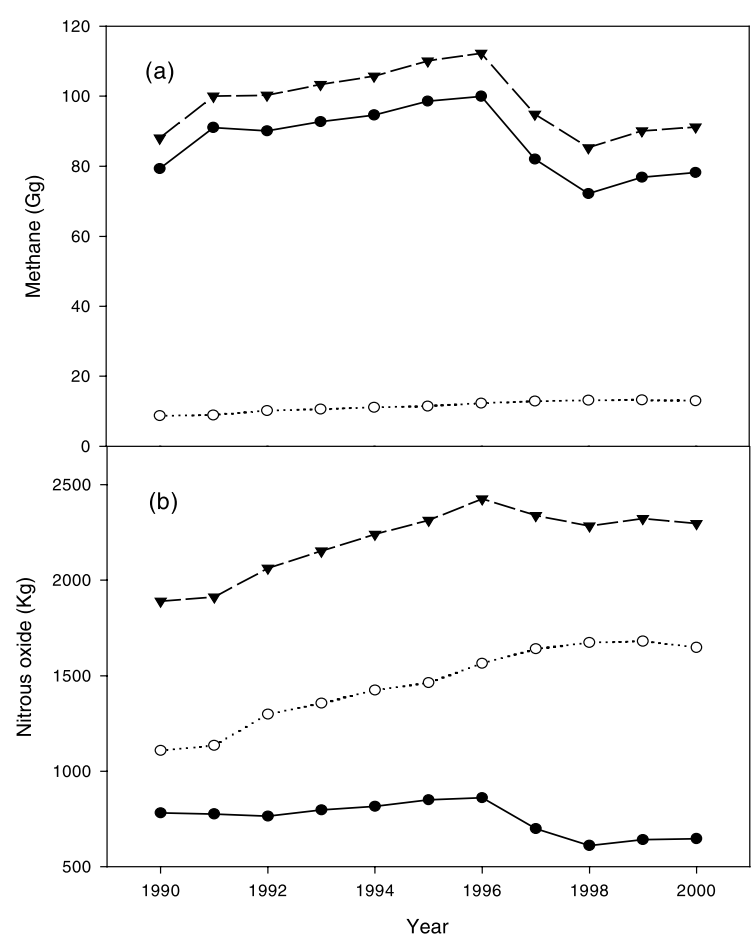

Fig. 1. Greenhouse gases emission from animal feeding sector in Taiwan: (a) methane emission; (b) nitrous oxide emission; (•) livestock; $(\bigcirc)$ poultry; $(\boldsymbol{\nabla})$ total. the highest 2.43 ton in 1996 , and down to 2.30 ton in 2000. There is an urgent need to reduce greenhouse gas emissions from animal feeding sector and to recover and utilize methane from animal waste management, because of their impact on global warming.

\section{Acknowledgements}

The authors thank Professors Y.P. Wang, C.C. Chao, H.Y. Wang, C.M. Lai, C.F. Lee and S.D. Chang for their helpful assistances and comments, National Science Council (NSC 86-2621-P002-003) and Environmental Protection Administration (EPA-89-fall-03-145) of Republic of China for financial supports.

\section{References}

Chao, C.C., 2001. Emission and mitigation of nitrous oxide flux from the composting and application of livestock wastes. In: Yang, S.S. (Ed.), Flux and Mitigation of Greenhouse Gases (III). Global Change Research Center, Department of Agricultural Chemistry and Agriculture Exhibition Hall, National Taiwan University, Taipei, Taiwan, pp. 115127.

Cicerone, R.J., Oremland, R.S., 1988. Biogeochemical aspects of atmospheric methane. Global Biochemical Cycles 2, 299327.

Council of Agriculture/ROC, 2001. Agriculture Yearbook 2000. Taipei, Taiwan.

Department of Agriculture and Forest, Provincial Taiwan Government, 1991-2000. Agriculture Yearbook 1990-1999. Nantou, Taiwan.

Francisco, R.V., 1997. Greenhouse gas sources and sinks from Philippine agriculture, land use and forestry. In: Moya, T.B. (Ed.), Greenhouse Gas Emissions, Aerosols, Land Use and Cover Changes in Southeast Asia. The Environmental Research Institute, Chulalongkorn University, Bangkok, Thailand, pp. 7-17.

Hong, C.M., 1986a. Amount and physical and chemical properties of hog wastes. In: Treatment and Utilization of Hog Wastes. Taiwan Livestock Research Institute, Tainan, Taiwan.

Hong, C.M., 1986b. Anaerobic digestion and the construction of red-bag anaerobic fermentor. In: Treatment and Utilization of Hog Wastes. Taiwan Livestock Research Institute, Tainan, Taiwan.

Huang, D.C., Wang, H.Y., 2000. Greenhouse gases emissions from broiler feeding sector in Taiwan area. Journal of the Chinese Livestock Society 29, 65-75.

Intergovernmental Panel on Climate Change (IPCC), 1997. Guidelines for National Greenhouse Gas Inventories: Workbook and Reference Manual. Agriculture (Chapter 4) and Waste (Chapter 6). OECD, Paris, France.

Isermann, K., 1994. Agriculture's share in the emission of trace gases affecting the climate and some cause-oriented proposals for sufficiently reducing this share. Environment Pollution 83, 95-111. 
Khalil, M.A.K., Rasmussen, R.A., Wang, M.X., Ren, L., 1991. Methane emission from rice fields in China. Environmental Sciences and Technology 25, 979-981.

Kinsman, R., Sauer, F.D., Jackson, H.A., Wolynetz, M.S., 1995. Methane and carbon dioxide emissions from dairy cows in full lactation monitored over a six-month period. Journal of Dairy Sciences 78, 2760-2766.

Lee, C.F., Shiao, T.F., Chen, C.P., 2001. Enteric methane production of Holstein dry cows and growing Heifers in Taiwan. In: Yang, S.S. (Ed.), Flux and Mitigation of Greenhouse Gases (III). Global Change Research Center, Department of Agricultural Chemistry and Agriculture Exhibition Hall, National Taiwan University, Taipei, Taiwan, pp. 157-172.

Lee, C.F., Shiao, T.F., Chen, C.P., Liu, S.C., 2000. Effect of dietary forage on milk performance and greenhouse gas production of Holstein lactation cows. In: Yang, S.S. (Ed.), Flux and Mitigation of Greenhouse Gases (II). Global Change Research Center, Department of Agricultural Chemistry and Agriculture Exhibition Hall, National Taiwan University, Taipei, Taiwan, pp. 205-225.

Lin, C.C., Chen, W.C., Chen, R.Y., 1989. Technical development of thermophilic fermentation in wastewater treatment of livestock. In: Proceedings of the Thermophilic Anaerobic Fermentation Technology. Institute of Industrial Technology, Hsinchu, Taiwan.

Lindau, C.W., Bollich, P.K., DeLaune, R.D., Mosier, A.R., Bronson, K.F., 1993. Methane mitigation in flooded Louisiana rice field. Biology and Fertility of Soils 136, 195-203.

Liu, C.M., Yang, S.S., Hong, C.C., 1996. Estimation of methane and nitrous oxide emissions in Taiwan area in 1990. Journal of the Biomass Energy Society of China 15, $1-8$.
Wong, C.C., Wu, A.T., Chen, Y.H., 1999. Development and prospects of the resource re-utilization of agriculture and livestock wastes. In: Proceedings of the Second Extension and Research in Resource Re-utilization of Livestock Wastes. Department of Agriculture and Forest of Provincial Taiwan Government, Taichung, Taiwan, pp. 7-21.

Yang, S.S., 1994. Livestock production and environment. In: Agriculture and Environment Conservation. Hua-ShangYuan Publisher, Taipei, Taiwan, pp. 119-143.

Yang, S.S., 1997. Greenhouse gas emissions from agriculture in Taiwan. In: Moya, T.B. (Ed.), Greenhouse Gas Emissions, Aerosols, Land Use and Cover Changes in Southeast Asia. The Environmental Research Institute, Chulalongkorn University, Bangkok, Thailand, pp. 26-35.

Yang, S.S., 1998. Methane production of river and lake sediments in Taiwan. Environmental Geochemistry and Health 18, 245-250.

Yang, S.S., Chang, H.L., 2001. Effect of green manure amendment and flooding on methane emission from paddy fields. Chemosphere, Global Change Science 3, 41-49.

Yang, S.S., Lin, C.F., Lin, H.C., 1996. Wastes Treatment and Re-Utilization. National Open University, Taipei, Taiwan. pp. 300 .

Yang, S.S., Lin, C.F., Wang, C.K., 2003. Wastes Treatment and Re-Utilization, second ed. National Open University, Taipei, Taiwan.

Yang, S.S., Liu, C.M., Lai, C.M., Liu, Y.L. Estimation of methane and nitrous oxide emission from rice paddy and upland in Taiwan during 1990-2000. Chemosphere (in press).

Yang, S.S., Wei, C.B., Koo, K., Tsai, S.S., 1991. Food and agricultural wastes produced in Taiwan area. Journal of the Biomass Energy Society of China 10, 70-87. 